\title{
Análise de Custos em uma Instituição de Ensino Superior: um Estudo Aplicado no Instituto Federal do Tocantins
}

\author{
Samara Oliveira Alves \\ Tecnologia em Logística pelo Instituto Federal do Tocantins - IFTO \\ 310 Sul, Av. LO 5, s/n. Plano Diretor Sul. Palmas/TO. CEP: 77021-090 \\ E-mail: alvessamara.oliveira.96@gmail.com \\ William Brasil Rodrigues Sobrinho \\ Mestrado em Ciências Contábeis pela Universidade Federal do Espírito Santo - UFES \\ Professor do Instituto Federal do Tocantins - IFTO \\ 310 Sul, Av. LO 5, s/n. Plano Diretor Sul. Palmas/TO. CEP: 77021-090 \\ E-mail:william@ifto.edu.br
}

\section{RESUMO}

O objetivo deste trabalho corresponde à aplicação de um modelo de gestão de custos por meio da utilização da premissa do método Custeio Baseado em Atividades (ABC) em um campus do Instituto Federal do Tocantins (IFTO), mediante um estudo de caso. Optou-se pela utilização do método $A B C$ em razão da vasta recomendação pela literatura existente. Constatou-se que cerca de $85 \%$ dos custos são referentes a folha de pagamentos dos servidores. Adicionalmente, identificou-se que somente $18,4 \%$ dos recursos são alocados em atividades de apoio ao ensino, enquanto $81,6 \%$ dos recursos são destinados às atividades primárias da instituição, ou seja, atividades diretamente relacionadas ao ensino, pesquisa e extensão. Por fim, o custo anual médio do aluno matriculado no IFTO campus Porto Nacional no ano de 2017 equivale a $\mathrm{R} \$ 16.596$.

Palavras-chaves: Gestão de Custo. Instituição de Ensino. Custeio Baseado em Atividades (ABC).

\section{A Graduate School Cost Analysis: an Applied Study in the Federal Institute of Tocantins}

\section{ABSTRACT}

This paper aims to check the application of a cost management model, through the use of the premise of the Activity Based Costing (ABC) method in a case study carried out in the campus of the Federal Institute of Tocantins (IFTO). The ABC method was chosen because of its vast recommendation in the existing literature. It was found that around $85 \%$ of the costs refer to servers payroll, and $18.4 \%$ of the resources are allocated to teaching support activities, while $81.6 \%$ of the resources are allocated to the institution's primary activities, i.e. teaching-related activities. Finally, the study presents an average 
Análise de Custos em uma Instituição de Ensino Superior: um Estudo Aplicado no Instituto Federal do Tocantins

Samara Oliveira Alves, William Brasil Rodrigues Sobrinho

of an annual cost of the student enrolled at IFTO in Porto Nacional campus in 2017, closed to $R \$ 16,596$.

Keywords: Cost Management. Educational institution. Activity-Based Costing (ABC).

\section{Análisis de Costos en una Institución de Educación Superior: un Estudio Aplicado en el Instituto Federal de Tocantins}

\section{RESUMEN}

El objetivo de este trabajo corresponde a la aplicación de un modelo de gestión de costos, utilizando la premisa del método de Costeo Basado en Actividades (ABC) en un campus del Instituto Federal de Tocantins (IFTO), através de un estudio de caso. Se opto por el uso del método $\mathrm{ABC}$ debido a la gran recomendación en la literatura existente. Se encontró que alrededor del $85 \%$ de los costos se refieren a la nómina de los servidores. Además, se identificó que uno $18.4 \%$ de los recursos se asignan a actividades de apoyo a la enseñanza, mientras uno $81.6 \%$ de los recursos se destinan a las actividades principales de la institución, es decir, actividades directamente relacionadas con la enseñanza, la investigación y la extensión. Finalmente, el costo anual promedio del estudiante matriculado en el IFTO en el Campus Porto Nacional en 2017 es equivalente a $\mathrm{R} \$ 16.596$.

Palabras clave: Gestión de costos. Institucion de enseñanza. Costeo basado en actividades $(\mathrm{ABC})$.

\section{INTRODUÇÃO}

Cabe ao gestor público o gerenciamento dos interesses da coletividade e compete a ele desenvolver ações direcionadas ao planejamento, direção, fiscalização, acompanhamento e controle dos projetos, atividades e programas governamentais. Essas ações possuem a finalidade de garantir o cumprimento das metas preestabelecidas no plano de governo e, em especial, quanto aos aspectos da economicidade, eficiência, eficácia e efetividade (Araújo \& Arruda, 2009).

O orçamento público é o instrumento no qual o administrador público dispõe para melhor aplicar os recursos financeiros. A despesa pública, em outras palavras, o compromisso dos gastos dos recursos governamentais, tem o objetivo de atender as 
Análise de Custos em uma Instituição de Ensino Superior: um Estudo Aplicado no Instituto Federal do Tocantins

Samara Oliveira Alves, William Brasil Rodrigues Sobrinho

necessidades de interesse coletivo previstas nos diversos instrumentos normativos aplicáveis ao setor público, tais como a Constituição da República Federativa do Brasil, a Lei no 4.320/64 e a Lei Complementar no 101/00.

Nesse âmbito, a gestão de custos no setor público pode ser utilizada como meio para se mensurar o valor do que se gasta na esfera pública, além de permitir análises mais detalhadas sobre a eficiência dos serviços prestados e os resultados alcançados e, ainda, permite a população saber quais foram os benefícios gerados a partir da aplicação de determinado recurso. O tema passou a ser visto com mais relevância para a sociedade, como uma forma objetiva de medir a eficiência da aplicação dos recursos arrecadados por meio dos impostos. Os cidadãos estão procurando averiguar mais detalhadamente a qualidade dos serviços concedidos (Cordeiro \& Alves, 2016).

No ano de 2005, por meio da Lei Federal o 11.195/2005, iniciou-se o plano de expansão da rede federal de educação profissional o que culminou em uma maior descentralização da atuação da rede federal e, consequentemente, maiores gastos públicos. Com base na necessidade de disponibilizar informações mais relevantes sobre os serviços prestados para a sociedade, questiona-se qual é a estrutura de custos necessária para se manter uma instituição federal de ensino na cidade de Porto Nacional? O objetivo do trabalho consiste em aplicar o método de custeio baseado em atividades ( $\mathrm{ABC}$ ) no campus da cidade de Porto Nacional do Instituto Federal do Tocantins (IFTO).

O objetivo relaciona-se com as profundas reestruturações que o setor público sofreu em seus serviços e, especialmente, em sua gestão. O Estado passou a almejar uma gestão mais próxima ao modelo encontrado no setor privado, por meio da adoção de lógicas de mercado e modelos de gestão nos quais a ênfase passa a ser o resultado. Essa reestruturação, oriunda da denominada Nova Gestão Pública (NGP), trouxe para o setor público características e práticas gerenciais empresariais, tais como a descentralização administrativa, artefatos de planejamento estratégico, gestão de custos, controladoria entre outros (Messias, Ferreira, \& Soutes, 2018). 
Análise de Custos em uma Instituição de Ensino Superior: um Estudo Aplicado no Instituto Federal do Tocantins Samara Oliveira Alves, William Brasil Rodrigues Sobrinho

Essa preocupação com os gastos fundamenta-se no atual contexto econômico de grandes transformações e contingenciamento financeiro das instituições públicas. Por sua vez, devem-se atentar ao fato da necessidade de um controle mais sistemático dos seus gastos (Silva, Francisco, Cruz, \& Bueno, 2016).

Visto a importância para a sociedade brasileira do conhecimento dos custos dos serviços prestados e da existência de indicadores para a avaliação dos benefícios das decisões governamentais. O presente estudo realiza uma análise mais detalhada dos custos incorridos em uma instituição de ensino superior do Tocantins e posteriormente possibilita aos gestores da instituição informações suplementares sobre a gestão dos custos.

\section{REVISÃO DE LITERATURA}

Segue a apresentação de um breve contexto sobre o custeio baseado em atividades $(A B C)$ e na sequência são elencados estudos anteriores realizados sobre a aplicação do custeio $A B C$ no setor público, em especial nas Instituições de Ensino Superior.

\subsection{Custeio Baseado em Atividades (ABC)}

É um método de custeio que procura reduzir as distorções provocadas pelo rateio arbitrário dos custos indiretos. $O$ custeio $A B C$ se apoia no planejamento, na execução e na mensuração do custo das atividades (Martins, 2010). O método ABC também é visto como sendo uma metodologia que viabiliza o tratamento de dados relacionado aos custos e as atividades que mais implicam dispêndio de recursos em uma empresa (Nakagawa, 2001). Vale destacar que as atividades são encontradas em todas as organizações, inclusive naquelas sem fins lucrativos, diante disso, torna-se possível utilizar o sistema $A B C$ em empresas não industriais, instituições financeiras e concessionárias de serviços públicos, pois atividades ocorrem tanto em processos de 
Análise de Custos em uma Instituição de Ensino Superior: um Estudo Aplicado no Instituto Federal do Tocantins Samara Oliveira Alves, William Brasil Rodrigues Sobrinho

manutenções financeiras quanto em concessionários de serviços públicos (Martins, 2010).

Segundo Slomski (2007), o custeio baseado em atividade (ABC) é o sistema de maior aplicabilidade ao setor público, pois na metodologia $A B C$ não são os produtos que consomem recursos, mas sim, as atividades e as ações desenvolvidas. Diante disso, o primeiro passo para $\circ \mathrm{ABC}$ consiste em identificar as atividades relevantes dentro de cada departamento (Santos, 2011).

O sistema de custos do setor público brasileiro deve buscar a melhoria na aplicação dos recursos enfatizando o processo de accountability (prestação de contas), propiciando aos stakeholders (partes interessadas) as informações sobre o desenvolvimento das suas atividades (Dantas \& Crozatti, 2014). Favorecendo assim, segundo a gestão, facilitar a análise de resultados (Leoncine, Bornia, \& Abbas, 2013). Para isso, é importante a estruturação do sistema de custos com todos os setores envolvidos no processo de apuração para que a administração tome as decisões com informações mais precisas (Araújo, Carneiro, \& Santana, 2015).

Em 2011, houve a implantação do Sistema de Informações de Custo do Governo Federal (SIC), o qual é operacionalizado pelos órgãos centrais e não pelas unidades finalistas. Diferentemente do que sugere a teoria institucional e a experiência internacional, o governo brasileiro implantou de uma solução tecnológica, em detrimento de uma mobilização institucional (Borges, Mario, \& Carneiro, 2013). No trabalho de Amaral, Bolfe, Borgert e Vicente (2014), por meio da aplicação de um survey, os autores identificaram que a maioria dos contadores das IFES - Instituições Federais de Ensino Superior não acreditam na possibilidade de o SIC obter as informações físico-financeiras dos sistemas estruturantes e não considera que a estrutura orçamentária existente obtenha os custos diretos dos serviços públicos.

Castro (2018) faz uma comparação da diferença entre o sistema de custos no setor público e no setor privado. No privado, é fundamental que os gestores conheçam os custos dos produtos ou serviços para o estabelecimento do preço, capaz de produzir 
Análise de Custos em uma Instituição de Ensino Superior: um Estudo Aplicado no Instituto Federal do Tocantins

Samara Oliveira Alves, William Brasil Rodrigues Sobrinho

o retorno do capital aplicado. Já no setor público, não é esse o foco e sim na solução de problemas identificados e também não está em jogo a sobrevivência do capital.

\subsection{Estudos Anteriores sobre Custos no Setor Público}

A produção científica sobre custos no setor público de acordo Söthe, Dill, Gubiani e Silva (2014) é crescente, embora os periódicos ainda apresentem escassa produção nacional sobre o tema (Alves, Martins, \& Martins, 2017). Estudos demonstram que o método $\mathrm{ABC}$ pode contribuir para um melhor processo decisório acerca da aplicação eficiente dos recursos públicos em instituições públicas, bem como em organizações hospitalares (Morais, Santos, \& Neto, 2019; Raimundini, Souza, Struett, \& Botelho, 2004; Struett \& Souza, 2002). Enquanto isso há uma tendência de se adotar o sistema de custeio por atividades pelo fato do orçamento ser elaborado por funções de governo e permitir maior accountability e economicidade (Moura, Cheibub, \& Costa Neto, 2001; Reis, Ribeiro, \& Slomski, 2005).

No que tange as Instituições Federais de Ensino Superior (IFES) há uma preocupação em relação ao indicador custo por aluno, principalmente em razão da criação pelo Tribunal de Contas da União (TCU), no ano de 2002, de um portfólio de indicadores para as IFES (Fereira Santos, \& Pessanha, 2013), o que despertou o interesse de autores pelo tema.

O estudo de Amaral (2004) constatou um redução do custo do aluno das IFES no período de 1995 a 2001. Enquanto Silva et al. (2004) desenvolve uma metodologia para o cálculo do custo aluno na Universidade de Brasília (UNB), que pode ser aplicada as demais instituições. O trabalho de Magalhães, Silveira, Abrantes, Ferreira e Wakim (2010) utilizou a sistemática do custeio por absorção com a finalidade de apurar o custo médio por aluno na Universidade Federal de Viçosa (UFV).

No tocante ao custo-médio por aluno das instituições pertencentes à rede federal de educação profissional, científica e tecnológica (EPCT), o trabalho de Pereira (2019) analisou a evolução do custo-médio por aluno dessas instituições no período de 2009 a 2016. O autor constatou que o valor relativo ao custo-médio por aluno sofreu acréscimo 
Análise de Custos em uma Instituição de Ensino Superior: um Estudo Aplicado no Instituto Federal do Tocantins

Samara Oliveira Alves, William Brasil Rodrigues Sobrinho

no período de 2009 a 2014. Nos anos seguintes 2015 e 2016, o custo-médio por aluno, dessas instituições, apresentaram redução de 25,7\% (2014-2015) e 11,5\% (20152016).

Por outro lado, os estudos também passaram a se preocupar com a gestão dos custos nas IFES. O trabalho de Peter et al. (2003), por exemplo, propõe um modelo de apuração e gestão de custos, fundamentado no Activity Based Costing (ABC) para a Universidade Federal do Ceará (UFC). O modelo consiste na separação das atividades em dois principais grupos (primárias e secundárias) de acordo com sua relação direta ou indireta com o ensino, pesquisa e a extensão. Silva et al. (2016) utiliza o método do custeio baseado em atividades ( $A B C)$ na Universidade Federal de Minas Gerais (UFMG), partindo da definição de cinco atividades básicas (ensino, pesquisa, extensão, gestão e suporte).

Por fim, o estudo de caso de Bentes (2015) utiliza como base a metodologia do custeio baseado em atividades para mensurar o custo por aluno no Instituto Federal do Amazonas (IFAM). Contudo, a metodologia proposta pelo autor enfatiza mais o indicador custo/aluno do que a gestão dos custos da instituição pelo método ABC.

\section{METODOLOGIA}

A pesquisa consiste na aplicação inicial do método $A B C$ no Instituto Federal do Tocantins (IFTO) no campus da cidade de Porto Nacional localizado à $60 \mathrm{~km}$ da capital do Tocantins, Palmas. O campus Porto Nacional do IFTO, surgiu na conjuntura da expansão da rede Federal de Educação, Ciência e Tecnologia por meio da Portaria no 102 de 29 de janeiro de 2010 do Ministério da Educação publicada no Diário Oficial da União de $1^{\circ}$ de fevereiro de 2010 (IFTO, 2010).

O IFTO - Campus Porto Nacional iniciou suas atividades em agosto de 2010 oferecendo dois cursos, sendo um de Graduação de Licenciatura em Computação e o outro em Tecnologia em Logística e, ainda, dois cursos subsequentes: um em Técnico em Informática e outro em Técnicos em Logística. Em 2012, passou a oferecer o curso 
Análise de Custos em uma Instituição de Ensino Superior: um Estudo Aplicado no Instituto Federal do Tocantins

Samara Oliveira Alves, William Brasil Rodrigues Sobrinho

Técnico em Meio Ambiente integrado ao ensino médio e o curso na modalidade do Programa Nacional de Integração da Educação Profissional com a Educação Básica na Modalidade de Jovens e Adultos (PROEJA) em Técnico em Vendas (IFTO, 2010). Atualmente oferece os cursos: Assistente Administrativo; Técnico em Administração; Técnico em Informática para Internet; Técnico em Meio Ambiente; Técnico em Informática; Técnico em Vendas, Licenciatura em Computação e Tecnologia em Logística.

\subsection{Método de Coleta dos Dados}

Os dados relacionados à execução orçamentária-financeira foram obtidos por meio das informações disponibilizadas ao público nos seguintes endereços eletrônicos: Portal da Transparência Fiscal - http://www.transparencia.gov.br/ e Instituto Federal do Tocantins - Campus Porto Nacional - TO - http://www.ifto.edu.br/porto.

As demais informações documentais foram obtidas nas seguintes instâncias administrativas do Campus: a) Gerência de Ensino; b) Gerência de Administração; c) Coordenação de Registros Escolares (CORES); d) Coordenação de Gestão de Pessoas; e) Coordenação Técnico-pedagógica (COTEPE) e f) Setor Financeiro e Orçamentário.

Os dados analisados são referentes à execução orçamentária-financeira do exercício fiscal de 2017 e correspondem a:

i) salários e benefícios (décimo terceiro, auxílio alimentação, auxílio moradia, auxílio saúde, gratificação natalina e outras gratificações) dos servidores da unidade;

ii) serviços de funcionamento e manutenção da unidade;

iii) material de consumo;

iv) manutenção predial;

v) equipamentos e materiais permanentes $e$

vi) despesas com oferecimento de benefícios e assistências aos estudantes. 
Análise de Custos em uma Instituição de Ensino Superior: um Estudo Aplicado no Instituto Federal do Tocantins Samara Oliveira Alves, William Brasil Rodrigues Sobrinho

\subsection{Obtenção e Tratamento dos Dados}

Para a obtenção dos dados necessários para elaboração do presente trabalho, foram realizadas as etapas a seguir:

1) Identificação das despesas orçamentárias do Campus, referente ao ano de 2017. As informações foram disponibilizadas pelo setor financeiro do Campus. Em seguida, com auxílio da administração foi possível identificar a estrutura física administrativa e algumas descrições do espaço físico da área do Campus. Logo foram identificadas as principais atividades de cada setor do Campus.

2) Coleta das áreas das salas que não foram informadas pela administração. Após a obtenção das áreas faltantes foram realizados os primeiros rateios, a partir da elaboração de um mapa de rateio das instalações do Campus. Com isso, realizaram-se os rateios das despesas orçamentárias do Campus de acordo as naturezas da despesa/áreas. As despesas foram alocadas nos setores conforme discriminado a seguir:

a. Despesas rateadas diretamente à Gerência de Administração: diárias no país, combustíveis e lubrificantes para outras finalidades, combustíveis e lubrificantes automotivos, ferramentas, material para comunicações, uniformes, tecidos e aviamentos, passagens para o país, serviço de seleção e treinamento, apoio administrativo técnico e operacional, serviços de telecomunicações, juros, restituições, serviços de publicidade legal, obras em andamento, máquinas e equipamentos energéticos, máquinas, utensílios e equipamentos diversos, mobiliário em geral.

b. Despesas rateadas de acordo com a área das instalações do Campus (por exemplo, coordenações, área de lazer, salas de aulas, laboratórios, ginásio e banheiros): limpeza e conservação, manutenção e conservação de bens imóveis, manutenção e conservação de bens móveis, serviços de água e esgoto, serviços de energia elétrica, contribuição para custeio de iluminação pública. 
Análise de Custos em uma Instituição de Ensino Superior: um Estudo Aplicado no Instituto Federal do Tocantins Samara Oliveira Alves, William Brasil Rodrigues Sobrinho

\section{c. As demais despesas foram alocadas nos seguintes departamentos: i)} Copa: gás e outros materiais engarrafados, material de copa e cozinha, serviços de copa e cozinha; ii) Coordenação de Pesquisa: auxílios para desenvolvimento de estudos e pesquisas, auxílio a pesquisadores; iii) Setor de Tecnologia da Informação: material de TIC (material de consumo), manutenção e conservação de equipamento de processamento de dados, material de TIC (permanente); iv) Coordenação de Assistência Estudantil: bolsas de estudo no país; v) Almoxarifado: material de expediente; vi) Laboratório de Química: material químico; vii) Laboratório de Biologia: sementes, mudas de plantas e insumos; viii) Enfermagem: material farmacológico, material hospitalar e ix) Reprografia: serviços de cópias e reprodução de documentos.

3) Aulas dos Docentes foram identificadas as quantidades semanais de aulas ministradas por cada docente do Campus. Essa informação foi obtida por meio dos horários de aulas. Com as informações da Coordenação de Gestão de Pessoas foi possível especificar os nomes, a atividade exercida, o setor de cada servidor do Campus e a sua respectiva remuneração. Foram identificados 106 servidores, dos quais 65 são docentes, desses, 7 encontravam-se em afastamento com recebimento de remuneração.

4) Custo da remuneração dos docentes foi rateada de acordo a quantidades de aulas ministradas por curso durante 0 ano de 2017. A remuneração dos demais servidores do Campus foi alocada de acordo com seus setores de atuação.

5) Elaboração de uma planilha que unificou os custos com servidores e as despesas orçamentárias, de acordo com as atividades de cada departamento. Nesse período também foram distinguidas todas as salas de aulas usadas de acordo com os cursos, ministrado no ano de 2017 , conforme informado pela Coordenação Técnico-pedagógica. 
Análise de Custos em uma Instituição de Ensino Superior: um Estudo Aplicado no Instituto Federal do Tocantins

Samara Oliveira Alves, William Brasil Rodrigues Sobrinho

As atividades realizadas por cada setor do IFTO foram identificadas na Figura 1.

\begin{tabular}{|c|c|c|}
\hline Departamentos & Atividades Definidas & \\
\hline Direção Geral & Exercer a direção do Campus & \multirow{8}{*}{ 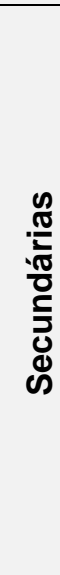 } \\
\hline $\begin{array}{l}\text { Coordenação de Gestão de } \\
\text { Pessoas }\end{array}$ & $\begin{array}{l}\text { Controlar a folha de pagamento e processos relacionados } \\
\text { ao pessoal }\end{array}$ & \\
\hline $\begin{array}{l}\text { Coordenação de Orçamento e } \\
\text { Finanças }\end{array}$ & Executar a administração financeira e orçamentária & \\
\hline $\begin{array}{l}\text { Coordenação de Pesquisa e } \\
\text { Inovação }\end{array}$ & $\begin{array}{l}\text { Integrar o Campus com as empresas e comunidade } \\
\text { externa }\end{array}$ & \\
\hline Gerência de Administração & Gerenciar a Administração do Campus & \\
\hline $\begin{array}{l}\text { Coordenação de Almoxarifado e } \\
\text { Patrimônio }\end{array}$ & Controlar o patrimônio e almoxarifado do Campus & \\
\hline $\begin{array}{l}\text { Coordenação de Tecnologia da } \\
\text { Informação }\end{array}$ & Controlar a tecnologia da informação do Campus & \\
\hline Setor de Protocolo & Expedir processos & \\
\hline Gerência de Ensino & Gerenciar o Ensino & \\
\hline $\begin{array}{l}\text { Coordenação do Curso de Meio } \\
\text { Ambiente Integrado ao Ensino } \\
\text { Médio }\end{array}$ & $\begin{array}{l}\text { Coordenar as atividades do ensino médio técnico em } \\
\text { meio ambiente }\end{array}$ & \\
\hline $\begin{array}{l}\text { Coordenação do Curso de } \\
\text { Informática Integrado ao Ensino } \\
\text { Médio }\end{array}$ & $\begin{array}{l}\text { Coordenar as atividades do ensino médio técnico em } \\
\text { informática para internet }\end{array}$ & \\
\hline $\begin{array}{lll}\text { Coordenação dos } & \text { Cursos } \\
\text { Técnicos Subsequentes } & \\
\end{array}$ & Coordenar as atividades do ensino técnico subsequente & \\
\hline $\begin{array}{lcc}\text { Coordenação } & \text { do Curso } & \text { de } \\
\text { Administração } & \text { Integrado } & \text { ao } \\
\text { Ensino Médio } & & \\
\end{array}$ & $\begin{array}{l}\text { Coordenar as atividades do ensino médio técnico em } \\
\text { administração }\end{array}$ & \\
\hline $\begin{array}{l}\text { Coordenação do Curso Superior } \\
\text { de Licenciatura em Computação }\end{array}$ & $\begin{array}{l}\text { Coordenar as atividades do curso superior de licenciatura } \\
\text { em computação }\end{array}$ & \\
\hline $\begin{array}{l}\text { Coordenação do Curso Superior } \\
\text { de Tecnologia em Logística }\end{array}$ & $\begin{array}{l}\text { Coordenar as atividades do curso superior de tecnologia } \\
\text { em logística }\end{array}$ & \\
\hline $\begin{array}{|ll|}\text { Coordenação } & \text { Técnico } \\
\text { Pedagógica (COTEPE) } & \\
\end{array}$ & Auxiliar no processo pedagógico & \\
\hline $\begin{array}{ll}\text { Coordenação Geral do } \\
\text { Complexo Laboratorial }\end{array}$ & Coordenar as atividades laboratoriais do Campus & \\
\hline Coordenação da Biblioteca & Controlar o acervo e empréstimo de livros & \\
\hline \begin{tabular}{|lr} 
Coordenação de & Registros \\
Escolares (CORES) & \\
\end{tabular} & Emitir e registrar diplomas & \\
\hline $\begin{array}{l}\text { Coordenação de Assistência Ao } \\
\text { Estudante }\end{array}$ & Assistir aos estudantes do Campus & \\
\hline
\end{tabular}

Figura 1. Atividades Definidas por Departamentos do IFTO - Campus Porto Nacional

Fonte: Elaboração dos autores (2018) com base nos dados do IFTO - Campus Porto Nacional. 
Análise de Custos em uma Instituição de Ensino Superior: um Estudo Aplicado no Instituto Federal do Tocantins

Samara Oliveira Alves, William Brasil Rodrigues Sobrinho

As atividades realizadas por cada setor do IFTO foram identificadas levando em consideração as principais atribuições do setor. A classificação das atividades, por setor, está representada na Figura 1.

Após a identificação das principais atividades de cada setor foi possível estabelecer o direcionamento necessário para realizar o rateio dos custos às atividades definidas. Posteriormente, as atividades foram classificadas em primárias e secundárias de acordo com a relação existente entre cada atividade e a finalidade da instituição. Nesse aspecto, as atividades primárias são aquelas relacionadas diretamente com o ensino, pesquisa ou extensão (Peter et al., 2003).

\subsection{Rateio dos custos por atividades definidas}

A alocação dos custos por atividades foi realizada seguindo critérios estabelecidos na Figura 2. Logo após foram consideradas como custo das atividades primárias todos os custos dos setores que estão ligados diretamente com o ensino, a pesquisa e a extensão, enquanto os demais custos foram relacionados pelas atividades de apoio.

(continuação)

\begin{tabular}{|l|l|}
\hline \multicolumn{1}{|c|}{ Atividades } & \multicolumn{1}{|c|}{ Custos Relacionados } \\
\hline $\begin{array}{l}\text { Exercer a Direção do } \\
\text { Campus }\end{array}$ & $\begin{array}{l}\text { Todos os custos com remuneração dos servidores lotados no setor e os } \\
\text { demais custos rateados pela direção geral. }\end{array}$ \\
\hline $\begin{array}{l}\text { Controlar a folha de } \\
\text { pagamento e processos } \\
\text { relacionados ao pessoal }\end{array}$ & $\begin{array}{l}\text { Todos os custos com remuneração dos servidores lotados no setor e os } \\
\text { demais custos rateados pela coordenação de gestão de pessoas. }\end{array}$ \\
\hline $\begin{array}{l}\text { Executar a administração } \\
\text { financeira e orçamentária }\end{array}$ & $\begin{array}{l}\text { Todos os custos com remuneração dos servidores lotados no setor e os } \\
\text { demais custos rateados pelo setor de orçamento e finanças. }\end{array}$ \\
\hline $\begin{array}{l}\text { Integrar o Campus com as } \\
\text { empresas e a comunidade } \\
\text { externa }\end{array}$ & $\begin{array}{l}\text { Todos os custos com remuneração dos servidores lotados no setor e os } \\
\text { demais custos rateados pela coordenação de integração empresa-escola- } \\
\text { comunidade e a coordenação de pesquisa e coordenação de extensão. }\end{array}$ \\
\hline $\begin{array}{l}\text { Gerenciar a administração } \\
\text { do Campus }\end{array}$ & $\begin{array}{l}\text { Todos os custos com remuneração dos servidores lotados no setor e os } \\
\text { demais custos rateados pela copa, banheiros feminino/ masculino e } \\
\text { banheiro/vestiário portadores de necessidades especiais, reprografia, sala } \\
\text { de áudio visual, ginásio poliesportivo, campo de futebol society, sala de } \\
\text { reuniões, áreas de vivência e a sala de apoio ao terceirizado. }\end{array}$ \\
\hline
\end{tabular}


(conclusão)

\begin{tabular}{|c|c|}
\hline Atividades & Custos Relacionados \\
\hline $\begin{array}{l}\text { Controlar o patrimônio e } \\
\text { almoxarifado do Campus }\end{array}$ & $\begin{array}{l}\text { Todos os custos com remuneração dos servidores lotados no setor e os } \\
\text { demais custos rateados pelo almoxarifado. }\end{array}$ \\
\hline $\begin{array}{l}\text { Controlar a tecnologia da } \\
\text { informação do Campus }\end{array}$ & $\begin{array}{l}\text { Todos os custos com remuneração dos servidores lotados no setor e os } \\
\text { demais custos rateados pela coordenação de tecnologia da informação. }\end{array}$ \\
\hline Expedir processos & $\begin{array}{l}\text { Todos os custos com remuneração dos servidores lotados no setor e os } \\
\text { demais custos rateados pelo setor de protocolo. }\end{array}$ \\
\hline Gerenciar o ensino & $\begin{array}{l}\text { Todos os custos com remuneração dos servidores lotados no setor e } \\
\text { custos com docentes afastados, sala dos professores, cabines de estudo } \\
\text { dos docentes, sala de descanso dos docentes e todas as salas de aulas } \\
\text { vazias. }\end{array}$ \\
\hline $\begin{array}{l}\text { Coordenar as atividades } \\
\text { do ensino médio técnico } \\
\text { em meio ambiente }\end{array}$ & $\begin{array}{l}\text { Todos os custos com remuneração dos docentes de acordo com a } \\
\text { quantidade de aulas ministradas nesse curso, demais custos rateados pela } \\
\text { coordenação do curso de meio ambiente integrado ao ensino médio e das } \\
\text { salas utilizadas pelo curso. }\end{array}$ \\
\hline $\begin{array}{l}\text { Coordenar as atividades } \\
\text { do ensino médio técnico } \\
\text { em informática para } \\
\text { internet }\end{array}$ & $\begin{array}{l}\text { Todos os custos com remuneração dos docentes de acordo com a } \\
\text { quantidade de aulas ministradas neste curso, demais custos rateados pela } \\
\text { coordenação do curso de informática integrado ao ensino médio e das } \\
\text { salas utilizadas pelo curso. }\end{array}$ \\
\hline $\begin{array}{l}\text { Coordenar as atividades } \\
\text { do ensino técnico } \\
\text { subsequente }\end{array}$ & $\begin{array}{l}\text { Todos os custos com remuneração dos docentes de acordo com a } \\
\text { quantidade de aulas ministradas neste curso, demais custos rateados pela } \\
\text { coordenação dos cursos técnicos subsequentes e das salas utilizadas pelo } \\
\text { curso. }\end{array}$ \\
\hline $\begin{array}{l}\text { Coordenar as atividades } \\
\text { do ensino médio técnico } \\
\text { em administração }\end{array}$ & $\begin{array}{l}\text { Todos os custos com remuneração dos docentes de acordo com a } \\
\text { quantidade de aulas ministradas neste curso, demais custos rateados pela } \\
\text { coordenação do curso de administração integrado ao ensino médio e das } \\
\text { salas utilizadas pelo curso. }\end{array}$ \\
\hline
\end{tabular}

Figura 2. Custos Relacionados pelas Atividades

Fonte: Elaboração dos autores (2018) com base nos dados do IFTO - Campus Porto Nacional.

A complexidade da atuação docente e a ausência de informações gerenciais são fatores dificultam a utilização dos direcionadores de custos, por isso o trabalho não os apresenta. Diante disso, uma das alternativas para o rateio dos valores relativos aos docentes afastados foi sua alocação para a atividade de gerenciar o ensino em razão da não atuação dos mesmos em outras atividades da instituição. 
Análise de Custos em uma Instituição de Ensino Superior: um Estudo Aplicado no Instituto Federal do Tocantins Samara Oliveira Alves, William Brasil Rodrigues Sobrinho

\section{APRESENTAÇÃO E ANÁLISE DOS RESULTADOS}

Desse modo, apresenta-se a composição geral dos custos da instituição relativa ao exercício financeiro de 2017 e, na sequência, realiza-se a análise dos resultados.

A análise dos resultados é dividida em três etapas: sendo que a primeira consiste na apresentação detalhada das atividades em separado (primárias e secundárias); já a segunda, verifica-se a consolidação das atividades e, finalmente, executa-se o cálculo do custo médio do aluno por duas abordagens distintas:

a) por atividade correspondente a cada curso ofertado pela instituição e;

b) no geral.

\subsection{Mapa dos Custos Gerais do IFTO - Campus Porto Nacional}

A Tabela 1 demonstra a composição do custo anual relativo ao ano de 2017 da instituição, contendo os valores gastos com manutenção, despesas com pessoal e de capital. O custo com pessoal e encargos representa $85 \%$ do gasto total da instituição de ensino, o qual consome grande parte do orçamento do IFTO - Campus Porto Nacional. Em um estudo similar, feito por Pessoa (2000) foi constatado que, o custo com pessoal das universidades federais tem consumido a quase totalidade dos recursos aportados pelo Tesouro, comprometendo o montante direcionado a outros custeios e capital. 
Análise de Custos em uma Instituição de Ensino Superior: um Estudo Aplicado no Instituto Federal do Tocantins Samara Oliveira Alves, William Brasil Rodrigues Sobrinho

Tabela 1

Mapa do Custo Geral do IFTO - Campus Porto Nacional (2017)

\begin{tabular}{|c|c|c|}
\hline Classificação & Valor em $\mathbf{R} \$$ & AV\% \\
\hline Pessoal e Encargos & 11.849 .508 & $85,00 \%$ \\
\hline Locação de Mão-de-Obra & 940.493 & $6,70 \%$ \\
\hline Auxílio Financeiro a Estudantes & 472.808 & $3,40 \%$ \\
\hline Outros Serviços de Terceiros - Pessoa Jurídica & 382.231 & $2,70 \%$ \\
\hline Material de Consumo & 118.862 & $0,90 \%$ \\
\hline Equipamento e Material Permanente & 70.097 & $0,50 \%$ \\
\hline Obras e Instalações & 46.585 & $0,30 \%$ \\
\hline Diárias - Pessoal Civil & 22.153 & $0,20 \%$ \\
\hline Passagens e Despesas com Locomoção & 17.166 & $0,10 \%$ \\
\hline Indenizações e Restituições & 11.433 & $0,10 \%$ \\
\hline Auxílio Financeiro a Pesquisadores & 4.620 & $0,00 \%$ \\
\hline Outros Serviços de Terceiros - Pessoa Física & 1.453 & $0,00 \%$ \\
\hline Obrigações Tributárias e Contributivas & 2.120 & $0,00 \%$ \\
\hline Outros Serviços de Terceiros - Pessoa Jurídica (Intra) & 1.108 & $0,00 \%$ \\
\hline Total & 13.940 .637 & $100 \%$ \\
\hline
\end{tabular}

Nota. Fonte: Elaboração dos autores (2018) com base nos dados do IFTO - Campus Porto Nacional.

Os demais custos relevantes do Campus Porto Nacional-IFTO são direcionados à locação de mão de obra, que corresponde a $6,7 \%$ do total, concessão de auxílio financeiro a estudantes $(3,4 \%)$ e outros serviços de terceiros - pessoa jurídica $(2,7 \%)$ do custo total. Por fim, os gastos com material de consumo, material permanente, obras e demais custos somados representam apenas $2,2 \%$ do montante.

\subsection{Custeio Geral do IFTO - Campus Porto Nacional por Meio de Uma Premissa Baseada em Atividades}

Os custos das atividades de apoio a administração da instituição são apresentados na Tabela 2. Os dados foram organizados em ordem de relevância do gasto. É possível observar que, com exceção da atividade "gerenciar a administração do Campus", o custo com pessoal representa a quase totalidade do custo de cada atividade. 
Análise de Custos em uma Instituição de Ensino Superior: um Estudo Aplicado no Instituto Federal do Tocantins

Samara Oliveira Alves, William Brasil Rodrigues Sobrinho

Tabela 2

Custo por Atividades de Apoio e Administração do IFTO - Campus Porto Nacional

\begin{tabular}{lrrrr}
\hline \multicolumn{1}{c}{ Atividades de Apoio } & Pessoal & \multicolumn{1}{c}{$\begin{array}{c}\text { Outros } \\
\text { Custos }\end{array}$} & $\begin{array}{c}\text { Total dos } \\
\text { Custos }\end{array}$ & AV\% \\
\hline Gerenciar a administração do Campus & 243.209 & 1.220 .015 & 1.463 .224 & 56,9 \\
Controlar a tecnologia da informação do Campus & 233.259 & 35.459 & 268.718 & 10,5 \\
Exercer a direção do Campus & 198.364 & 5.375 & 203.740 & 7,9 \\
$\begin{array}{l}\text { Controlar a folha de pagamento e processos } \\
\text { relacionados ao pessoal }\end{array}$ & 177.554 & 2.693 & 180.247 & 7,0 \\
$\begin{array}{l}\text { Integrar o Campus com as empresas e comunidade } \\
\text { externa }\end{array}$ & 164.721 & 14.099 & 178.820 & 7,0 \\
$\begin{array}{l}\text { Executar a administração financeira e orçamentária } \\
\text { Expedir processos }\end{array}$ & 106.390 & 2.693 & 109.083 & 4,2 \\
Controlar o patrimônio e almoxarifado do Campus & 85.478 & 1.346 & 86.824 & 3,4 \\
\hline $\begin{array}{l}\text { Total das Atividades de Apoio e Administração do } \\
\text { Campus }\end{array}$ & $\mathbf{1 . 2 8 1 . 8 6 3}$ & $\mathbf{1 . 2 8 9 . 0 8 7}$ & $\mathbf{2 . 5 7 0 . 9 5 0}$ & $\mathbf{1 0 0}$ \\
\hline
\end{tabular}

Nota. Fonte: Elaboração dos autores (2018) com base nos dados do IFTO - Campus Porto Nacional.

Conforme sugestão de Peter et al. (2003), as atividades foram classificadas em secundárias e primárias. Neste trabalho as atividades de apoio foram consideradas atividades secundárias. Nesse sentido, constatou-se que dos $\mathrm{R} \$ 13,9$ milhões executados no período, $R \$ 2,6$ milhões foram direcionados às atividades de apoio, o que representa $18,4 \%$ da execução financeira no ano de 2017. Em outras palavras, a maior parte da execução financeira foi realizada por meio das atividades consideradas primárias (de ensino), as quais serão analisadas em sequência. Esse percentual de $18,4 \%$ relativo à prática das atividades de apoio é próximo ao encontrado em trabalhos anteriores (Peter et al., 2003; Silva et al., 2016).

A atividade de gerenciar a administração do Campus constitui aproximadamente $57 \%$ dos custos das atividades de apoio. Nesse aspecto reside uma das limitações do presente trabalho, por questões administrativas e organizacionais da instituição, a maior parcela dos outros custos foi alocada para a atividade gerenciar a administração. Isso se deve à dificuldade e, em alguns casos, à impossibilidade de direcionar determinado custo à outra atividade. A atividade de controlar a tecnologia de informação do Campus 
Análise de Custos em uma Instituição de Ensino Superior: um Estudo Aplicado no Instituto Federal do Tocantins

Samara Oliveira Alves, William Brasil Rodrigues Sobrinho

representa 10,5\% das atividades secundárias. As demais atividades de apoio possuem o seu custo influenciado basicamente pelo custo com pessoal.

$\mathrm{Na}$ Tabela 3 são demonstrados os custos das atividades primárias, ou seja, àquelas vinculadas diretamente com o ensino. As atividades primárias totalizaram aproximadamente $\mathrm{R} \$ 11,4$ milhões correspondendo a $81,6 \%$ da execução financeira do ano em análise.

Tabela 3

Custo por Atividades Primárias do IFTO - Campus Porto Nacional

\begin{tabular}{lrrrr}
\hline \multicolumn{1}{c}{ Atividades Primárias } & Pessoal & \multicolumn{1}{c}{$\begin{array}{c}\text { Outros } \\
\text { Custos }\end{array}$} & \multicolumn{1}{c}{$\begin{array}{c}\text { Total dos } \\
\text { Custos }\end{array}$} & AV\% \\
\hline Coordenar o curso de licenciatura em computação & 1.970 .133 & 29.680 & 1.999 .814 & 17,6 \\
Coordenar o ensino médio técnico em meio ambiente & 1.304 .328 & 17.995 & 1.322 .323 & 11,6 \\
Coordenar o curso superior de tecnologia em logística & 1.290 .514 & 21.688 & 1.312 .201 & 11,5 \\
Coordenar o ensino médio técnico em informática para & 1.140 .200 & 25.835 & 1.166 .035 & 10,3 \\
internet & 1.128 .499 & 35.470 & 1.163 .969 & 10,2 \\
Gerenciar o Ensino & 1.092 .790 & 25.294 & 1.118 .084 & 9,8 \\
Coordenar o ensino médio técnico em administração & 619.945 & 477.645 & 1.097 .590 & 9,7 \\
Assistir aos estudantes do Campus & 668.619 & 9.061 & 677.680 & 6,0 \\
Coordenar o ensino técnico subsequente & 555.214 & 4.171 & 559.385 & 4,9 \\
Auxiliar no processo pedagógico & 441.013 & 34.111 & 475.124 & 4,2 \\
Controlar o acervo e empréstimo de livros & 297.547 & 5.375 & 302.922 & 2,7 \\
Emitir e registrar diplomas & 58.842 & 115.717 & 174.559 & 1,5 \\
Coordenar as atividades laboratoriais do Campus & $\mathbf{1 0 . 5 6 7 . 6 4 5}$ & $\mathbf{8 0 2 . 0 4 3}$ & $\mathbf{1 1 . 3 6 9 . 6 8 8}$ & $\mathbf{1 0 0}$ \\
\hline Total das Atividades Relacionadas ao Ensino do & & & & \\
Campus & & &
\end{tabular}

Nota. Fonte: Elaboração dos autores (2018) com base nos dados do IFTO - Campus Porto Nacional.

A atividade de coordenar o curso superior de licenciatura em computação representa $17,6 \%$ do custo relativo das atividades primárias. Em sequência, a atividade de coordenar o ensino médio técnico em meio ambiente representa $11,6 \%$ do custo das atividades primárias, seguido da atividade de coordenar o curso superior de tecnologia em logística a qual representa $11,5 \%$ dos custos das atividades primárias. Coordenar o ensino médio técnico em informática para internet representa $10,3 \%$ dos custos das 
Análise de Custos em uma Instituição de Ensino Superior: um Estudo Aplicado no Instituto Federal do Tocantins

Samara Oliveira Alves, William Brasil Rodrigues Sobrinho

atividades primárias, essas quatro atividades juntas representam $51 \%$ do custo total das atividades primárias. Há um maior esforço da instituição em alocação de recursos para essas quatro atividades, provavelmente devido a maior necessidade de recursos humanos para desenvolver atividades nessas áreas. Cursos de tecnologia, como os de informática, exigem mais recursos das instituições, especialmente no que tange a infraestrutura física de laboratórios e equipamentos de informática. A Tabela 4 explana a consolidação dos custos das atividades.

Tabela 4

Custo Geral do IFTO - Campus Porto Nacional - Segregado por Atividade

\begin{tabular}{lrc}
\hline \multicolumn{1}{c}{ Atividades de Apoio } & Total dos Custos & AV\% \\
\hline Gerenciar a administração do Campus & 1.463 .224 & 10,5 \\
Controlar a tecnologia da informação do Campus & 268.718 & 1,9 \\
Exercer a direção do Campus & 203.740 & 1,5 \\
Controlar a folha de pagamento e processos relacionados ao pessoal & 180.247 & 1,3 \\
Integrar o Campus com as empresas e comunidade externa & 178.820 & 1,3 \\
Executar a administração financeira e orçamentária & 109.083 & 0,8 \\
Expedir processos & 86.824 & 0,6 \\
Controlar o patrimônio e almoxarifado do Campus & 80.294 & 0,6 \\
\hline Subtotal das Atividades de Apoio e Administração do Campus & $\mathbf{2 . 5 7 0 . 9 5 0}$ & $\mathbf{1 8 , 4}$ \\
\hline & & \\
Atividades Primárias & Total dos Custos & $\mathbf{A V \%}$ \\
\hline Coordenar o curso superior de licenciatura em computação & 1.999 .814 & 14,3 \\
Coordenar as atividades do ensino médio técnico em meio ambiente & 1.322 .323 & 9,5 \\
Coordenar as atividades do curso superior de tecnologia em logística & 1.312 .201 & 9,4 \\
Coordenar o ensino médio técnico em informática para internet & 1.166 .035 & 8,4 \\
Gerenciar o Ensino & 1.163 .969 & 8,3 \\
Coordenar as atividades do ensino médio técnico em administração & 1.118 .084 & 8,0 \\
Assistir aos estudantes do Campus & 1.097 .590 & 7,9 \\
Coordenar as atividades do ensino técnico subsequente & 677.680 & 4,9 \\
Auxiliar no processo pedagógico & 559.385 & 4,0 \\
Controlar o acervo e empréstimo de livros & 475.124 & 3,4 \\
Emitir e registrar diplomas & 302.922 & 2,2 \\
Coordenar as atividades laboratoriais do Campus & 174.559 & 1,3 \\
\hline Subtotal das Atividades Relacionadas ao Ensino do Campus & $\mathbf{1 1 . 3 6 9 . 6 8 8}$ & $\mathbf{8 1 , 6}$ \\
\hline TOTAL GERAL & $\mathbf{1 3 . 9 4 0 . 6 3 7}$ & $\mathbf{1 0 0}$ \\
\hline
\end{tabular}

Nota. Fonte: Elaboração dos autores (2018) com base nos dados do IFTO - Campus Porto Nacional. 
Análise de Custos em uma Instituição de Ensino Superior: um Estudo Aplicado no Instituto Federal do Tocantins Samara Oliveira Alves, William Brasil Rodrigues Sobrinho

Essa apresentação é relevante para melhor entendimento da composição dos custos por atividades da instituição de forma agregada.

De forma consolidada foi possível identificar que coordenar as atividades do curso superior de licenciatura em computação representa $14,3 \%$ dos custos gerais do instituto. Em seguida gerenciar a administração do Campus equivale a 10,5\% do montante. Constata-se que a maior parte dos custos dos Campus estão direcionados para as atividades primárias, ou seja, aquelas destinadas diretamente ao ensino, semelhante a alguns trabalhos anteriores (Peter et al., 2003; Silva et al., 2016).

A vantagem de se comparar os custos das atividades de apoio (administração) com as atividades que estão diretamente relacionadas com o ensino é a possibilidade de se propor mudanças que podem contribuir ainda mais para melhorar a efetividade da organização (Peter et al., 2003).

Complementarmente foi realizado o cálculo do custo médio do aluno por duas abordagens diferentes: i) pelo custo da respectiva atividade primária de cada curso e ii) geral.

Na primeira metodologia, adotou-se apenas os gastos das atividades primárias específicas de cada curso da instituição. Por exemplo, para a atividade coordenar as atividades do curso superior de licenciatura em computação, foi levado em consideração apenas o quantitativo de alunos matriculados no respectivo curso superior para determinar o custo/aluno. Para as demais atividades seguiu-se o mesmo critério. Ressalva-se que nesta abordagem os valores de custos por aluno não consideram as demais atividades.

Diante disso é apresentado na Tabela 5 o custo médio das atividades primárias relacionadas aos cursos ofertados pela instituição, ponderada pelas matrículas efetivadas no ano calendário de 2017. Ressalta-se que nesta abordagem não é levado em consideração as demais atividades geradoras de custo, mas somente a atividade primária específica de cada curso. A utilização do número de alunos matriculados como um direcionador dos custos das atividades dos cursos de graduação, nas Instituição 
Análise de Custos em uma Instituição de Ensino Superior: um Estudo Aplicado no Instituto Federal do Tocantins

Samara Oliveira Alves, William Brasil Rodrigues Sobrinho

Federais de Ensino Superior, possibilita transformar o montante de custos dos cursos em um indicador comparável entre cursos: o custo do aluno (Cordeiro \& Alves, 2016).

Tabela 5

Custo por Alunos das Atividades Primárias Relacionadas aos Cursos Oferecidos Pelo Campus Porto Nacional

\begin{tabular}{lccc}
\hline \multicolumn{1}{c}{ Atividades Primárias Relacionadas aos Cursos } & $\begin{array}{c}\text { Custo das } \\
\text { Atividades }\end{array}$ & Matrículas & $\begin{array}{c}\text { Custo Médio das } \\
\text { Atividades }\end{array}$ \\
\hline $\begin{array}{l}\text { Coordenar as Atividades do Ensino Técnico } \\
\text { Subsequente }\end{array}$ & 677.680 & 58 & 11.786 \\
$\begin{array}{l}\text { Coordenar as Atividades do Ensino Médio Técnico } \\
\text { em Meio Ambiente }\end{array}$ & 1.322 .323 & 117 & 11.350 \\
$\begin{array}{l}\text { Coordenar as Atividades do Ensino Médio Técnico } \\
\text { em Informática para Internet }\end{array}$ & 1.166 .035 & 104 & 11.212 \\
$\begin{array}{l}\text { Coordenar as Atividades do Ensino Médio Técnico } \\
\text { em Administração }\end{array}$ & 1.118 .084 & 112 & 9.983 \\
$\begin{array}{l}\text { Coordenar as Atividades do Curso Superior de } \\
\text { Licenciatura em Computação }\end{array}$ & 1.999 .814 & 224 & 8.948 \\
$\begin{array}{l}\text { Coordenar as Atividades do Curso Superior de } \\
\text { Tecnologia em Logística }\end{array}$ & 1.312 .201 & 227 & 5.793 \\
\hline Total & $\mathbf{7 . 5 9 6 . 1 3 8}$ & $\mathbf{8 4 0}$ & $\mathbf{9 . 0 4 3}$ \\
\hline
\end{tabular}

Nota. Fonte: IFTO - Campus Porto Nacional - TO, com elaboração dos autores (2018).

Em contrapartida aqueles cursos com um número menor de matrículas ao longo do ano-calendário tendem a resultar em um custo médio por aluno mais elevado. Diante disso, o cálculo do custo médio anual por aluno da instituição é apresentado na Tabela 6. 
Análise de Custos em uma Instituição de Ensino Superior: um Estudo Aplicado no Instituto

Federal do Tocantins

Samara Oliveira Alves, William Brasil Rodrigues Sobrinho

Tabela 6

Custo Por Aluno - Geral do IFTO - Campus Porto Nacional

\begin{tabular}{lcccc}
\hline \multicolumn{1}{c}{ No de Matrículas } & $\mathbf{2 0 1 7 / 1}$ & $\mathbf{2 0 1 7 / 2}$ & Média & AV\% \\
\hline Curso Superior de Tecnologia em Logística & 217 & 236 & 227 & 27,0 \\
Curso Superior de Licenciatura em Computação & 221 & 226 & 224 & 26,6 \\
Curso Ensino Médio Técnico em Meio Ambiente & 118 & 115 & 117 & 13,9 \\
Curso Técnico Ensino Médio Administração & 113 & 111 & 112 & 13,3 \\
Curso Ensino Médio Técnico em Informática para & 104 & 104 & 104 & 12,4 \\
Internet & 37 & 30 & 34 & 4,0 \\
Curso Ensino Técnico Subsequente em Informática & 19 & 29 & 24 & 2,9 \\
Curso Ensino Técnico Subsequente em Vendas & $\mathbf{8 2 9}$ & $\mathbf{8 5 1}$ & $\mathbf{8 4 0}$ & $\mathbf{1 0 0 \%}$ \\
\hline Total de Matrículas & & & 1.383 & 0,01 \\
Custo Médio Mensal do Estudante & & & $\mathbf{1 3 . 9 4 0 . 6 3 7}$ & $\mathbf{1 0 0 \%}$ \\
Custo Médio Anual do Estudante & & & & \\
\hline Custo Total do Ano & & & &
\end{tabular}

Nota. Fonte: IFTO - Campus Porto Nacional - TO, com elaboração dos autores (2018).

Considerando uma média de 840 matrículas ao longo de 2017 têm-se um custo médio anual por estudante de $\mathrm{R} \$ 16.596$.

Na pesquisa de Amaral (2004), o autor estimou o custo aluno das IFES no valor de $R \$ 5.488$ para o ano de 2001 ( $R \$ 14.982$ em valores corrigidos pelo IPCA, a preços de dez/2017), no estudo de Silva et al. (2004) realizado na Universidade de Brasília os autores identificaram que o custo aluno para o ano de 2003 foi de $R \$ 5.187$ ( $R \$ 11.498$ em valores corrigidos pelo IPCA, a preços de dez/2017).

Enquanto o trabalho de Magalhães et al. (2010) apresenta um custo médio por aluno no valor de $R \$ 8.966$ para 2004 na Universidade Federal de Viçosa ( $R \$ 18.533$ em valores corrigidos pelo IPCA, a preços de dez/2017). No estudo de Pereira (2019), o custo médio por aluno das instituições da rede federal no ano de 2016 foi estimado em $\mathrm{R} \$ 11.867$ ( $\mathrm{R} \$ 12.218$ em valores corrigidos pelo IPCA, a preços de dez/2017) e no trabalho de Bentes (2015), o autor estimou um custo aluno no valor de $R \$ 30.177$ para o ano de 2014 ( $\mathrm{R} \$ 38.885$ em valores corrigidos pelo IPCA, a preços de dez/2017).

Conforme Magalhães et al. (2010), as experiências de apuração de custo por aluno nas instituições de ensino superior costumam apresentar resultados diferentes. Essas diferenças podem ser explicadas devido à localização geográfica da instituição de ensino, pois as instituições descentralizadas tendem a possuir uma estrutura de 
Análise de Custos em uma Instituição de Ensino Superior: um Estudo Aplicado no Instituto Federal do Tocantins

Samara Oliveira Alves, William Brasil Rodrigues Sobrinho

custos mais elevada (Høstmark-Tarrou, 1999), ou ainda, instituições com maior infraestrutura de apoio ao aluno possuem custo por aluno superior àquelas cuja infraestrutura de apoio é menor (Eicher, 2000).

\section{CONSIDERAÇÕES FINAIS}

O Custeio Baseado em Atividades ( $A B C$ ), embora tenha sido incialmente desenvolvido para a aplicação em indústrias, pode ser replicado para outros setores da economia e ainda pode ser adaptado à administração pública, com os devidos ajustes necessários (Mello, 2006). Na concepção de Peter, Martins, Pessoa e Peter (2003), o custeio baseado em atividades é considerado o método mais adequado para gestão de custos nas IFES (Instituições Federais de Ensino Superior), isso devido às suas peculiaridades tais como, a grande diversidade de produtos/serviços e a elevada estrutura de custos fixos.

O trabalho teve como objetivo aplicar o método de custeio baseado em atividades $(A B C)$ no Instituto Federal do Tocantins (IFTO) no Campus Porto Nacional. Com base no estudo de caso realizado foi possível identificar e classificar as principais atividades da instituição em primárias e secundárias. Assim sendo, constatou-se que a menor parcela dos recursos $18,4 \%$ é alocada em atividades secundárias, ou seja, atividades de apoio ao ensino. O restante $81,6 \%$ dos recursos são destinados as práticas de atividades primárias da instituição, ou seja, aquelas direcionadas diretamente ao ensino, pesquisa e extensão. O que é um fator positivo tendo em vista que a maior parte do esforço do Campus é destinado a atividade primárias.

Em outra análise realizada, embora sem considerar todos os custos da instituição, devido à restrição da metodologia adotada, identificou-se que o custo médio por aluno matriculado nos cursos do ensino técnico subsequente possui o maior valor ( $R \$ 11.786$ ), enquanto o custo médio por aluno do curso superior de tecnologia em logística possui o menor valor médio ( $\mathrm{R} \$ 5.793$ ). Fatores relativos ao baixo número de 
Análise de Custos em uma Instituição de Ensino Superior: um Estudo Aplicado no Instituto Federal do Tocantins

Samara Oliveira Alves, William Brasil Rodrigues Sobrinho

matrícula e duração do curso influenciam esses valores, pois quanto menor a quantidade de alunos matriculados em um curso, maior será o custo do curso para a instituição. Por fim, o custo anual médio do aluno matriculado na instituição consiste no valor de $\mathrm{R} \$ 16.596$ para o ano de 2017.

No que tange as limitações do presente estudo, ressalta-se que no processo de coletas dos dados foram encontradas algumas restrições nos setores devido à falta de sistemas/informações gerenciais de controle. Diante disso, uma parcela significativa dos custos foi rateada para a atividade gerenciar a administração. Isso ocorreu devido à dificuldade e, em alguns casos, a impossibilidade de identificar os direcionadores de custos adequados a cada atividade. Recomenda-se a adoção e otimização de sistemas de controle gerenciais para que a instituição de ensino disponha de dados futuros para mensuração de suas atividades, contribuindo assim para uma melhor análise futura.

Espera-se que os resultados obtidos, possam ser utilizados pelo IFTO - Campus Porto Nacional, em auxílio a decisões futuras, tais como: ampliar ou eliminar determinados projetos ou cursos, aumentar ou diminuir vagas dos cursos ofertados por essa instituição, melhorar a eficiência e eficácia tanto das atividades relacionadas ao ensino como as de apoio a administração da instituição e, principalmente, no processo de melhoria de alocação dos recursos orçamentários da instituição. Todavia, apesar dos esforços para uma maior exatidão das informações obtidas através da aplicação do sistema de apuração dos custos, por meio do método $A B C$, acredita-se que novos estudos podem ser feitos tanto para aumentar a exatidão das informações já obtidas como para servir de comparações anuais dessa mesma instituição.

Portanto, sugere-se que pesquisas futuras sejam realizadas no sentido de realizar a aplicação do método em Institutos Federais, tornando possível uma melhor comparação entre os resultados, pois a estrutura dos Institutos Federais difere das Universidades Federais, em especial, no que tange a oferta de cursos técnicos e de tecnologia. Além disso, cada ambiente possui as suas especificidades, por exemplo, os docentes atuam em ensino, pesquisa, extensão e administração, o que amplia a complexidade da abordagem e rastreabilidade dos custos. 
Análise de Custos em uma Instituição de Ensino Superior: um Estudo Aplicado no Instituto Federal do Tocantins Samara Oliveira Alves, William Brasil Rodrigues Sobrinho

\section{REFERÊNCIAS}

Alves, M. C., Martins, C. M. F., \& Martins, P. L. (2017). Custos no Setor Público: Reflexões sobre a incidência na literatura nacional veiculada em periódicos acadêmicos. Anais do Encontro Brasileiro de Administração Pública, 934-951.

Amaral, N. C. do. (2004). Evolução do custo do aluno das IFES: Eficiência? Avaliação: Revista da Avaliação da Educação Superior, 9(2), 115-125.

Amaral, N. A. de L., Bolfe, C., Borgert, A., \& Vicente, E. F. R. (2014). O Sistema de Custos do Governo Federal e as Responsabilidades dos Contadores das IFES. Revista de Contabilidade do Mestrado em Ciências Contábeis da UERJ, 19(2), 2339.

Araújo, C. A. de, Carneiro, A. de F., \& Santana, A. F. B. (2015). Sistemas de Custos Públicos: Entendimento e Implantação nos Municípios de Rondônia. Revista de Contabilidade do Mestrado em Ciências Contábeis da UERJ, 20(2), 66-84.

Araújo, I., \& Arruda, D. (2009). Contabilidade pública: Da teoria à prática (2a ed.). Saraiva.

Bentes, A. C. L. (2015). Um modelo de apuração de custos/aluno aplicado ao Instituto Federal de Educação, Ciência e Tecnologia do Amazonas-IFAM (Dissertação de Mestrado). Universidade Federal do Amazonas, UFAM, Amazonas, Brasil.

Borges, T. B., Mario, P. do C., \& Carneiro, R. (2013). A implementação do sistema de custos proposto pelo governo federal: Uma análise sob a ótica institucional. Revista de Administração Pública, 47(2), 469-491.

Castro, D. P. (2018). Auditoria, Contabilidade e Controle Interno no Setor Público (7a ed.). Atlas.

Cordeiro, J. F., \& Alves, T. (2016). Proposta de Modelo de Análise de Custos para Cursos de Graduação da área de Ciências Sociais Aplicadas em IFES. Congresso Brasileiro de Custos, ABC, Porto de Galinhas, PE, Brasil, 22.

Dantas, J. M. M., \& Crozatti, J. (2014). Implantação do sistema de custos no governo federal: Percepção da maturidade das setoriais de custos. Cadernos de Finanças Públicas, 14, 221-264.

Eicher, J.-C. (2000). The Financing of Education: An Economic Issue? European Journal of Education, 35(1), 33-44. 
Fereira, M. C., Santos, W. J. L. dos, \& Pessanha, J. F. M. (2013). Avaliação do Ensino Superior: Análise dos Indicadores Instituídos Pelo TCU Para as IFES. Revista de Contabilidade do Mestrado em Ciências Contábeis da UERJ, 18(1), 104-124.

Høstmark-Tarrou, A.-L. (1999). The Evaluation of Structures in European Universities. European Journal of Education, 34(3), 267-281.

IFTO. (2010). Projeto Pedagógico do Curso Superior de Tecnologia em Logística.

Leoncine, M., Bornia, A. C., \& Abbas, K. (2013). Sistemática para apuração de custos por procedimento médico-hospitalar. Produção, 23(3), 595-608.

Magalhães, E. A. de, Silveira, S. de F. R., Abrantes, L. A., Ferreira, M. A. M., \& Wakim, V. R. (2010). Custo do ensino de graduação em instituições federais de ensino superior: $O$ caso da Universidade Federal de Viçosa. Revista de Administração Pública, 44(3), 637-666.

Martins, E. (2010). Contabilidade de custos (10a ed.). Atlas.

Mello, G. S. de. (2006). Sistema de Custos na Administração Pública-Estudo da Aplicação do Método ABC em Prefeituras de Pequeno Porte. Congresso Brasileiro de Custos, Belo Horizonte, MG, Brasil, 13.

Messias, D., Ferreira, J. C., \& Soutes, D. O. (2018). Gestão de custos no setor público: Um panorama de experiências internacionais. Revista do Serviço Público, 69(3), 585-604.

Morais, G. M., Santos, V. F. dos, \& Neto, M. T. R. (2019). Gestão de custos no setor público: Um estudo em um restaurante universitário. Brazilian Journal of Development, 5(3), 1913-1933.

Moura, R. de A., Cheibub, T. P., \& Costa Neto, E. S. (2001). Gestão de Custos no Setor Público. Congresso Brasileiro de Custos, ABC, São Leopoldo, RS, Brasil, 8.

Nakagawa, M. (2001). ABC: Custeio baseado em atividades. Atlas.

Pereira, J. V. (2019). A Evolução do Gasto-Médio/Aluno e Custo-Médio/Aluno da Rede Federal de Educação Profissional, Científica e Tecnológica. Revista Brasileira de Política e Administração da Educação, 35(2), 329.

Pessoa, M. N. M. (2000). Gestão das universidades federais brasileiras: Um modelo fundamentado no balanced scorecard (Tese de Doutorado). Universidade Federal de Santa Catarina, UFSC, Santa Catarina, SC, Brasil. 
Peter, M. da G. A., Martins, E., Pessoa, M. N. M., \& Peter, F. A. (2003). Proposta de um Sistema de Custos Para as Universidades Federais Brasileiras Fundamentado no Activity Based Costing. Encontro da ANPAD - EnANPAD, Atibaia, SP, Brasil, 27.

Raimundini, S. L., Souza, A. A. de, Struett, M. A. M., \& Botelho, E. M. (2004). Aplicabilidade do Sistema $A B C$ e Análise de Custos Hospitalares: Comparação Entre Hospital Público e Hospital Privado. Encontro da ANPAD - EnANPAD, Curitiba, PR, Brasil, 28.

Reis, L. G. D., Ribeiro, P. A., \& Slomski, V. (2005). Custos no setor público: Uma proposta de implementação de sistemas de custeio. Congresso Brasileiro de Custos, ABC, Florianópolis, SC, Brasil, 9.

Santos, W. V. dos. (2011). Sistema de Informação de Custos do Governo Federal: Modelo Conceitual, Solução Tecnológica e Gestão do Sistema. Anais do Congresso CONSAD de Gestão Pública, Brasília, DF, Brasil, 4.

Silva, C. A. T., Morgan, B. F., \& Costa, P. de S. (2004). Desenvolvimento e aplicação de uma metodologia para cálculo do custo-aluno de instituições públicas de ensino superior: Um estudo de caso. Revista de Administração Pública, 38(2), 243-260.

Silva, E. R. S., Francisco, W. F. R., Cruz, D. B. da, \& Bueno, B. (2016). Custeio baseado em atividades: Uma proposta do seu uso no contexto de uma Universidade Federal de Minas Gerais. Congresso Brasileiro de Custos, ABC, Porto de Galinhas, PE, Brasil, 23.

Slomski, V. (2007). Controladoria e governança na gestão pública. Atlas.

Söthe, A., Dill, R. P., Gubiani, C. A., \& Silva, M. (2014). Custos no Setor Público: Análise da Inserção do Método ABC após a Publicação da LRF e do Estudo $\mathrm{n}^{\circ} 12$ do IFAC. Revista Estudo \& Debate, 21(1).

Struett, M. A. M., \& Souza, A. A. D. (2002). Análise da Aplicabilidade do Custeio Baseado Em Atividades Em Um Hospital Público. Congresso Brasileiro de Custos, ABC, São Paulo, SP, Brasil, 11.

Data de Submissão: 09/04/2019

Data de Aceite: 02/06/2020 